\title{
Ketone production by ketogenic diet and by intermittent fasting has different effects on the gut microbiota and disease progression in an Alzheimer's disease rat model
}

\author{
Sunmin Park,* Ting Zhang, Xuangao Wu, and Jing Yi Qiu \\ Department of Food and Nutrition, Obesity/Diabetes Research Center, Hoseo University, 165 Sechul-Ri Baebang-Yup, Asan-si, Chungchungnam-Do, 336795, Korea
}

(Received 24 September, 2019; Accepted 22 December, 2019; Published online 20 March, 2020)

\begin{abstract}
The benefits of ketone production regimens remain controversial. Here, we hypothesized that the ketone-producing regimens modulated cognitive impairment, glucose metabolism, and inflammation while altering the gut microbiome. The hypothesis and the mechanism were explored in amyloid- $\beta$ infused rats. Rats that received an amyloid- $\beta(25-35)$ infusion into the hippocampus had either ketogenic diet (AD-KD), intermittent fasting (AD-IMF), 30 energy percent fat diet (AD-CON), or high carbohydrate (starch) $\operatorname{diet}(A D-C H O)$ for 8 weeks. AD-IMF and AD-CHO, but not AD-KD, lowered the hippocampal amyloid- $\beta$ deposition compared to the $A D-C O N$ despite serum ketone concentrations being elevated in both $A D-K D$ and AD-IMF. AD-IMF and AD-CHO, but not AD-KD, improved memory function in passive avoidance, $Y$ maze, and water maze tests compared to the AD-CON. Hippocampal insulin signaling (pAkt $\rightarrow$ pGSK-3ß) was potentiated and pTau was attenuated in $A D-I M F$ and $A D-C H O$ much more than AD-CON. $A D-I M F$ and $A D-C O N$ had similar glucose tolerance results during OGTT, but AD-KD and AD-IMF exhibited glucose intolerance. AD-KD exacerbated gut dysbiosis by increasing Proteobacteria, and AD$\mathrm{CHO}$ improved it by elevating Bacteriodetes. In conclusion, ketone production itself might not improve memory function, insulin resistance, neuroinflammation or the gut microbiome when induced by ketone-producing remedies. Intermittent fasting and a high carbohydrate diet containing high starch may be beneficial for people with dementia.
\end{abstract}

Key Words: ketone, amyloid- $\beta$, hippocampus, insulin signaling, gut microbiome

A lzheimer's disease (AD) is a chronic neurodegenerative disease that results in $50-70 \%$ of dementia. The number of $\mathrm{AD}$ patients is increasing with increasing life expectancy since the incidence of AD increases rapidly in people over 70 years of age. ${ }^{(1)}$ Moreover, changing lifestyles and dietary patterns may be contributing to obesity, insulin resistance, and chronic inflammation that are believed to be risk factors for $\mathrm{AD}$, although their relationship to the etiology of AD remains poorly understood. ${ }^{(2)}$ AD progression is characterized by the accumulation of $\beta$-amyloid plaques and neurofibrillary tangles in the brain. ${ }^{(2)}$ Brain insulin resistance is also involved in $\mathrm{AD}$ progression, and it is accompanied by increased neuroinflammation and oxidative stress in the brain. $^{(3,4)}$ The increase of oxidative stress in AD is involved in the mitochondrial dysfunction that increases reactive oxygen species. ${ }^{(5)}$ Thus, oxidative stress, inflammation and insulin resistance in the brain can lead to the development of AD. These changes in the brain are reported to be connected to systemic insulin resistance. ${ }^{(6)}$
Obesity increases amyloid- $\beta$ deposition and neurofibrillary tangles, which promote the progression of $\mathrm{AD} .{ }^{(7)}$ The reduction of abdominal fat mass can be an intervention to prevent metabolic diseases, including Alzheimer's disease in the elderly, especially in post-menopausal women. Caloric restriction is known to be beneficial for the reduction of body fat, oxidative stress, inflammation, and cognitive dysfunction. ${ }^{(8,9)}$ However, it is difficult to reduce body fat by decreasing calorie intake, since people have difficulty with compliance for long periods. Various measures have been used by people for losing body fat. Ketogenic diets (KD) and intermittent fasting (IMF) are recommended to promote fat utilization as an energy source. People are restricted to consuming $50-100 \mathrm{~g}$ of carbohydrate per day with adequate protein intake in the $\mathrm{KD}$, whereas people can eat food only during certain periods in IMF. ${ }^{(10,11)}$ Both measures promote fat utilization in the body by making ketone bodies, which are products of incomplete oxidization of fatty acids. KD induces ketosis, but its effects on energy, glucose, and lipid metabolism are inconsistent. ${ }^{(12)}$ In our previous studies, ${ }^{(13,14)}$ IMF decreased epididymal fat pads by increasing energy expenditure in young rats but IMF, especially with HF diets, increased insulin resistance, possibly by attenuating hepatic insulin signaling. ${ }^{(13)}$ However, in AD-induced estrogendeficient rats, IMF protected against the deterioration of memory function and lipid metabolism in AD-induced estrogen-deficient rats. ${ }^{(14)}$ However, IMF might be associated with disturbances in the gut microbiome.

The gut is home to trillions of bacterial cells and thousands of bacterial species. ${ }^{(15)}$ Although the microbiota in adults is fairly resilient, it can be changed within individuals by both internal (disease status) and external (diets) stimuli. The availability of nutrients for gut microbiota modify composition the gut microbiome. Persons consuming high levels of microbiota-accessible carbohydrate (MAC) display greater microbial richness and diversity as compared to those consuming low levels of MAC. ${ }^{(16)}$ MAC restriction reduces short-chain fatty acid (SCFA) production, the primary end products of bacterial fermentation. The composition of SCFA plays an important role in altering body metabolism. High production of acetate is reported to increase insulin resistance through the gut-brain axis and high production of propionate and butyrate act as histone deacetylase inhibitors to epigenetically influence host gene expression. ${ }^{(17)}$ Thus, changes in the gut microbiome are linked to not only inflammation in the gut, but also energy, glucose, and lipid metabolisms.

KD change substrate utilization in intermediary metabolism and

*To whom correspondence should be addressed. E-mail: smpark@hoseo.edu 
increases the production of ketones for use as an energy source instead of carbohydrates. They can also change the gut microbiome. ${ }^{(18)}$ Children with severe epilepsy are sometimes treated with KD, and fiber-consuming bacteria becomes less abundant. ${ }^{(19)}$ Therefore, we hypothesized that interventions that increase blood ketone levels, including KD and IMF, may modulate memory function, and energy and glucose metabolisms in rats with memory impairment, and that they may also modify the gut microbiome. We examined the hypothesis in rats infused with amyloid$\beta$ into the hippocampus.

\section{Materials and Methods}

Animal care and surgical procedures. Male Sprague Dawley rats that weighed an average of $194 \pm 16 \mathrm{~g}$ were purchased from Daehan Bio, Inc. (Eum-Sung, Korea) and were acclimated in the animal facility. Rats were raised in individual stainless-steel cages in a controlled environment $\left(23^{\circ} \mathrm{C}, 12-\mathrm{h}\right.$ light/dark cycle) and they had free access to food and water. All study procedures were adopted based on the Guide for the Care and Use of Laboratory Animals from the National Institutes of Health and were approved by the Institutional Animal Care and Use Committee of Hoseo University (2014-06). After 1-week of acclimation in the animal facility, the rats were anesthetized with an intraperitoneal injection of a mixture of ketamine and xylazine (100 and $10 \mathrm{mg} / \mathrm{kg}$, respectively which was used for all procedures requiring anesthesia) and placed in a stereotaxic device. A cannula was inserted into the bilateral CA1 subregions of the hippocampus as previously described..$^{(20,21)}$ The cannula was connected to 22-gauge tubing filled with amyloid- $\beta(25-35)$ for the four $\mathrm{AD}$ groups or amyloid- $\beta(35-25)$ for the Non-AD-CON group; amyloid- $\beta(35-25)$ was used for the Non-AD-CON group as a control because it has the reverse sequence of amyloid- $\beta(25-35)$ and is not toxic and does not aggregate in the brain. ${ }^{(22)}$ Both types of amyloid- $\beta$ were dissolved in sterile saline and infused into the bilateral CA1 subregions using an osmotic pump (Alzet Osmotic Pump Company, Cupertino, CA) at a rate of $3.6 \mathrm{nmol} /$ day for 14 days. At the initial day of amyloid- $\beta$ infusion, the assigned diet was provided for each group for 8 weeks.

Diet preparation and experimental design. All rats had free access to a modified polyphenol-free AIN-93 semi-purified high-fat diet, which has been shown to induce obesity and insulin resistance. ${ }^{(13)}$ The normal diet was a modified semi-purified AIN-93 formulation consisting of 53 energy percent (En\%) carbohydrates, ${ }^{(23)}$ $19 \mathrm{En} \%$ protein, and $28 \mathrm{En} \%$ fats. The ketogenic diet was composed of $0.1 \mathrm{En} \%$ carbohydrates, $19 \mathrm{En} \%$ protein, and $81 \mathrm{En} \%$ fats, whereas the high $\mathrm{CHO}$ diet contained $68 \mathrm{En} \%$ carbohydrates, $19 \mathrm{En} \%$ protein, and $13 \mathrm{En} \%$ fats (Table 1). The major carbohydrate, protein, and fat sources were starch plus sugar $(2.4: 1, \mathrm{w} / \mathrm{w})$, casein (milk protein), and soybean oil plus lard (CJ Co., Seoul). The proper amounts of starch, casein, lard, and vitamin and mineral mixture were mixed, sifted, and stored at $4{ }^{\circ} \mathrm{C}$. The respective diet was provided by pressing the weighed powder tightly into the food container every other day, and the remaining feed was weighed and discarded.

The rats were divided into five treatment groups of 10 rats each: AD model rats that received either a $28 \%$ fat diet (normal diet) with ad libitum feeding (AD-CON), ketogenic diet (AD-KD), high $\mathrm{CHO}$ diet (AD-CHO), and normal diet with intermittent fasting (AD-IMF). The sham-operated rats received the same diet (normal diet) as AD-CON (Non-AD-CON).

Energy expenditure. Energy expenditure was measured by indirect calorimetry during the dark phase following $6 \mathrm{~h}$ of fasting at 3 days after the intraperitoneal insulin tolerance test. The rats were placed in a computer-controlled metabolic chamber with a computer-monitored $\mathrm{O}_{2}$ and $\mathrm{CO}_{2}$ system and $800 \mathrm{ml} / \mathrm{min}$ airflow (Biopac Systems Inc., Goleta, CA). The $\mathrm{CO}_{2}$ production $\left(\mathrm{VCO}_{2}\right)$ and $\mathrm{O}_{2}$ consumption $\left(\mathrm{VO}_{2}\right)$ of the rats were measured every minute for $30 \mathrm{~min}$, and the average $\mathrm{VO}_{2}$ and average $\mathrm{VCO}_{2}$ were integrated. The respiratory quotient (RQ) was calculated as $\mathrm{VCO}_{2} / \mathrm{VO}_{2}$ and $\mathrm{VO}_{2}$ and $\mathrm{VCO}_{2}$ values were adjusted for metabolic body size $\left(\mathrm{kg}^{0.75}\right){ }^{(13)}$ Resting energy expenditure and oxidations of fat and carbohydrate were calculated as previously described. ${ }^{(13)}$

Memory function by passive avoidance, $Y$ maze, and water maze tests. At the 7 th week, the rats were tested for memory deficits using a passive avoidance apparatus consisting of a two-compartment dark/light shuttle box. ${ }^{(13,24)}$ In the acquisition trial, electroshocks $(75 \mathrm{~V}, 0.2 \mathrm{~mA}, 50 \mathrm{~Hz})$ were delivered for $5 \mathrm{~s}$, immediately after the rats had entered the dark chamber. Five seconds later, the rat was removed from the dark chamber and returned to its home cage. After 24 and $48 \mathrm{~h}$, the retention latency time to enter the dark chamber was measured in the same way as in the acquisition trial, but electric foot shock was not delivered and the latency time was recorded to a maximum of $600 \mathrm{~s}$. Shorter latencies indicate memory deficit, compared to significantly longer latencies to enter the dark room.

The next day after the passive avoidance test, rats were subjected to the $\mathrm{Y}$ maze test which consisted of a horizontal maze with 3 arms at $120^{\circ}$ angles. Each arm was $50.5 \mathrm{~cm}$ in length, $20 \mathrm{~cm}$ in width, and $20 \mathrm{~cm}$ in height. Initially, rats were placed in one arm and then the sequences of entering into the other arms were monitored for $8 \mathrm{~min}$. A right alternation was defined as a rat consecutively entering into all three arms. When each arm in the $\mathrm{Y}$ maze was assigned as $\mathrm{A}, \mathrm{B}$, and $\mathrm{C}$, the right consecutive alternation was $\mathrm{ABC}, \mathrm{BCA}$, or $\mathrm{CAB}$, but not $\mathrm{CAC}, \mathrm{BAB}$, or $\mathrm{ABA}$. The spontaneous alternation (\%) was calculated from the following equation: $\%$ alternation $=[($ Number of right alternations $) /($ Total number of arm entries -2$)] \times 100$.

Spatial memory function was assessed with a Morris water maze test, as previously described, ${ }^{(22,24)}$ during day 49 . The Morris water maze tests hippocampal-dependent learning, including the acquisition of spatial memory. The platform was located in the

Table 1. The composition of experimental diets (Unit: $\mathrm{g} / \mathrm{kg}$ diet)

\begin{tabular}{lccc}
\hline & Normal diet & Ketogenic diet & High carbohydrate diet \\
\hline Corn starch & 371 & 1 & 470 \\
Sucrose & 200 & 1 & 200 \\
Casein & 210 & 320 & 190 \\
Lard & 115 & 574 & 36 \\
Soybean oil & 20 & 20 & 20 \\
Cellulose & 34 & 34 & 34 \\
AIN-76 mineral mixture & 35 & 35 & 35 \\
AIN-76 vitamin mixture & 10 & 10 & 10 \\
Choline chloride & 2 & 2 & 2 \\
DL-Methionine & 3 & 3 & 3 \\
Cholesterol & 25 & 25 & 25 \\
\hline
\end{tabular}


zone 5 of the water pool and rats started to swim from the zone 1. The longer period to find the platform indicated memory deficiency. The point for entry of the rat into the pool and the location of the platform for the escape remained unchanged for trials on days 1,2 , and 3 . On day 5 , the platform was removed and the time to go to zone 5 and staying in zone 5 were measured. The test was conducted with a cut-off time of $600 \mathrm{~s}$.

Glucose metabolism and sample collection. At week 8 of the experiment, an oral glucose tolerance test was conducted after an overnight fast using an oral administration of glucose $(2 \mathrm{~g} / \mathrm{kg})$. During the oral glucose tolerance test, serum glucose concentrations were measured every $10 \mathrm{~min}$ until $90 \mathrm{~min}$ and again at $120 \mathrm{~min}$, and serum insulin levels were measured at $0,20,40,60$, 90 , and $120 \mathrm{~min}$. The serum glucose and insulin concentrations were measured using a Glucometer (Accuchek; Roche Diagnostics, Basel, Switzerland) and enzyme-linked immunosorbent assay (ELISA; Crystal Chem, Elk Grove Village, IL), respectively. At 3 days after the oral glucose tolerance test, an intraperitoneal insulin tolerance test was conducted after the food was removed for $6 \mathrm{~h}$. The serum glucose levels were measured every $15 \mathrm{~min}$ for $90 \mathrm{~min}$ after an intraperitoneal injection of insulin $(0.75 \mathrm{U} / \mathrm{kg}$ body weight $)$.

After two days from the intraperitoneal insulin tolerance test, blood was collected from anesthetized rats by cardiac puncture, and the serum was separated by centrifugation at 3,000 rpm for $20 \mathrm{~min}$. After blood collection, human insulin $(5 \mathrm{U} / \mathrm{kg}$ body weight) was injected through the inferior vena cava to determine insulin signaling. Serum and tissue samples were stored at $-70^{\circ} \mathrm{C}$ for biochemical analysis. The epididymal and retroperitoneal fat masses and uteri were then removed and weighed. HOMA-IR was calculated as follows: serum insulin $(\mu \mathrm{U}) \times$ serum glucose $(\mathrm{mmol} / \mathrm{L}) / 22.5$. Serum tumor necrosis factor- $\alpha(\mathrm{TNF}-\alpha)$ concentrations were measured using ELISA kits from eBioscience (San Diego, CA).

Realtime quantitative reverse transcriptase polymerase chain reaction (RT-PCR). Hippocampal tissues were randomly excised from 4 rats from each group and total RNA was isolated using a monophasic solution of phenol and guanidine isothiocyanate (Trizol reagent; Invitrogen, Rockville, MD). The cDNA was synthesized from total RNA with superscript III reverse transcriptase and high fidelity Taq DNA polymerase $(1: 1: 1, \mathrm{v}: \mathrm{v}: \mathrm{v})$ by reverse transcription reaction in PCR. The synthesized cDNA was mixed with the primers of the genes of interest and sybergreen mix and their expressions were analyzed using a realtime PCR machine (BioRad Laboratories, Hercules, CA). The primers of TNF- $\alpha$, interleukin (IL)- $1 \beta$, and $\beta$-actin were given in previous studies. ${ }^{(13,22)}$ The expression levels genes of interest were quantified using the cycle of threshold method. ${ }^{(25)}$

Immunoblot analysis. Hippocampal tissues from four rats from each group were dissected as previously described. ${ }^{(22)}$ Each tissue was lysed with RIPA lysis buffer with added protease inhibitors, and their protein contents were measured using a BioRad protein assay kit (Hercules, CA). The lysates with equivalent amounts of protein $(30-50 \mu \mathrm{g})$ were resolved into sodium dodecyl sulfate-polyacrylamide gel electrophoresis and the amounts of proteins of interest were examined by immunoblotting with the specific antibodies as follows: protein kinase $\mathrm{B}$ (PKB or Akt), phosphorylated $\mathrm{PKB}^{\mathrm{Ser} 473}$, forkhead box protein $\mathrm{O} 1$ (FOXO1), phosphorylated FOXO1 ${ }^{\text {Ser256, }}$, phosphorylated tau ${ }^{\text {Ser396, }}$, tau (Cell Signaling Technology, Danvers, MA) and $\beta$-actin (Santa Cruz Biotech, Dallas, TX). The intensity of the proteins detected were measured using Imagequant TL (Amersham Biosciences, Piscataway, NJ).

Next-generation sequencing gut microbiome and bacterial sequence processing. The gut microbiome community was examined from the feces by analyzing metagenome sequencing using next-generation sequencing procedures. ${ }^{(26)}$ Bacterial DNA was extracted from the feces of each rat using a Power Water DNA Isolation Kit (Qiagen, Valencia, CA) according to the manufac- turer's instructions. The DNA was amplified with $16 \mathrm{~S}$ amplicon primers (V3 and V4 region) using PCR and each library was prepared using the PCR products according to the GS FLX plus library prep guide. ${ }^{(26)}$ The emulsion of DNA capture beads and library-beads was dispensed into a 96-well plate and the PCR amplification program was run with $16 \mathrm{~S}$ universal primers in the FastStart High Fidelity PCR System (Roche, Basel, Switzerland) according to the manufacturer's recommendations. Sequencing of bacterial DNA in the feces was measured by using the Illumina MiSeq standard operating procedure by a Genome Sequencer FLX plus (454 Life Sciences) in Macrogen Ltd. (Seoul, Korea).

The 16S amplicon sequences were processed using Mothur ver. 1.36. We followed the Miseq SOP to identify the taxonomy and counts of the bacteria in each fecal sample. We aligned the sequences using Silva reference alignment ver. 12350. In a preclustering step, the sequences with an identity $\geq 99 \%$ were merged. The chimeric sequences were detected and discarded by UCHIME. All the sequences were assigned with taxonomic classifications using Greengenes 13899 and the sequences classified as mitochondria, eukaryota or unknown were removed. We conducted the picking of operational taxonomic units (OTUs) delimited at $98 \%$ identity, which was taxonomically classified by consensus using Greengenes 13_8_99. A relaxed neighbor-joining tree with one representative sequence per OTU was obtained with Clearcut after calculating uncorrected pairwise distances between aligned reads.

Statistical analysis. All statistical analyses were conducted using SAS ver. 7 (SAS Institute, Cary, NC). All results are expressed as means \pm SD. One-way analysis of variance was used to compare the groups when the results were measured only once at the end of the experiment. Multiple comparisons among the groups were conducted using Tukey's test. Principle coordinate analysis (PCoA) in gut bacteria was analyzed by analysis of molecular variance in Mothur. A $p$ value $<0.05$ was considered as statistically significant.

\section{Results}

Cognitive function. Rats usually make right turns in the Y maze and then go to the next arm instead of going back to the previous arm. Rats in the AD-CON had a lower ratio of right turns to the total movements than the Non-AD-CON. Rats in the $\mathrm{AD}-\mathrm{IMF}$ and $\mathrm{AD}-\mathrm{CHO}$ had a higher ratio than $\mathrm{AD}-\mathrm{CON}$, and the ratio was similar to the Non-AD-CON (Fig. 1A).

In the 1st passive avoidance trial all rats entered into the darkroom and they had a small electric shock. Rats in the ADCON had a shorter latency compared to those in the Non-AD$\mathrm{CON}$, indicating that $\mathrm{AD}-\mathrm{CON}$ entered into the darkroom after forgetting the electric shock. AD-IMF delayed the latency to enter the darkroom in the 2nd trial compared to the other AD groups, and it was similar to Non-AD-CON (Fig. 1B). In 3rd trial rats in the AD-IMF and Non-AD-CON did not enter the darkroom. AD$\mathrm{CHO}$ increased the latency to enter the dark room but it was quicker than that of Non-AD-CON. AD-KD did not delay the latency compared to the AD-CON (Fig. 1B). Thus, AD-IMF was beneficial for enhancing short-memory and AD-CON had partial improvement.

In the 4th trial of the water maze test to determine the spatial memory, AD-CON rats showed a delayed latency time to locate the zone 5 where the platform was located in comparison to the Non-AD-CON (Fig. 1C). AD-KD did not improve the latency period to go to zone 5, and AD-IMF shortened the latency time to go to zone 5 as much as the Non-AD-CON. Interestingly, AD$\mathrm{CHO}$ went to zone 5 more quickly than the Non-AD-CON (Fig. 1C). During the 4 th water maze trial, the platform was removed, and rats were hunting for the platform. If rats remembered the platform area, they would stay longer in zone 5 . The duration in zone 5 was measured. Rats in the AD-CON stayed 

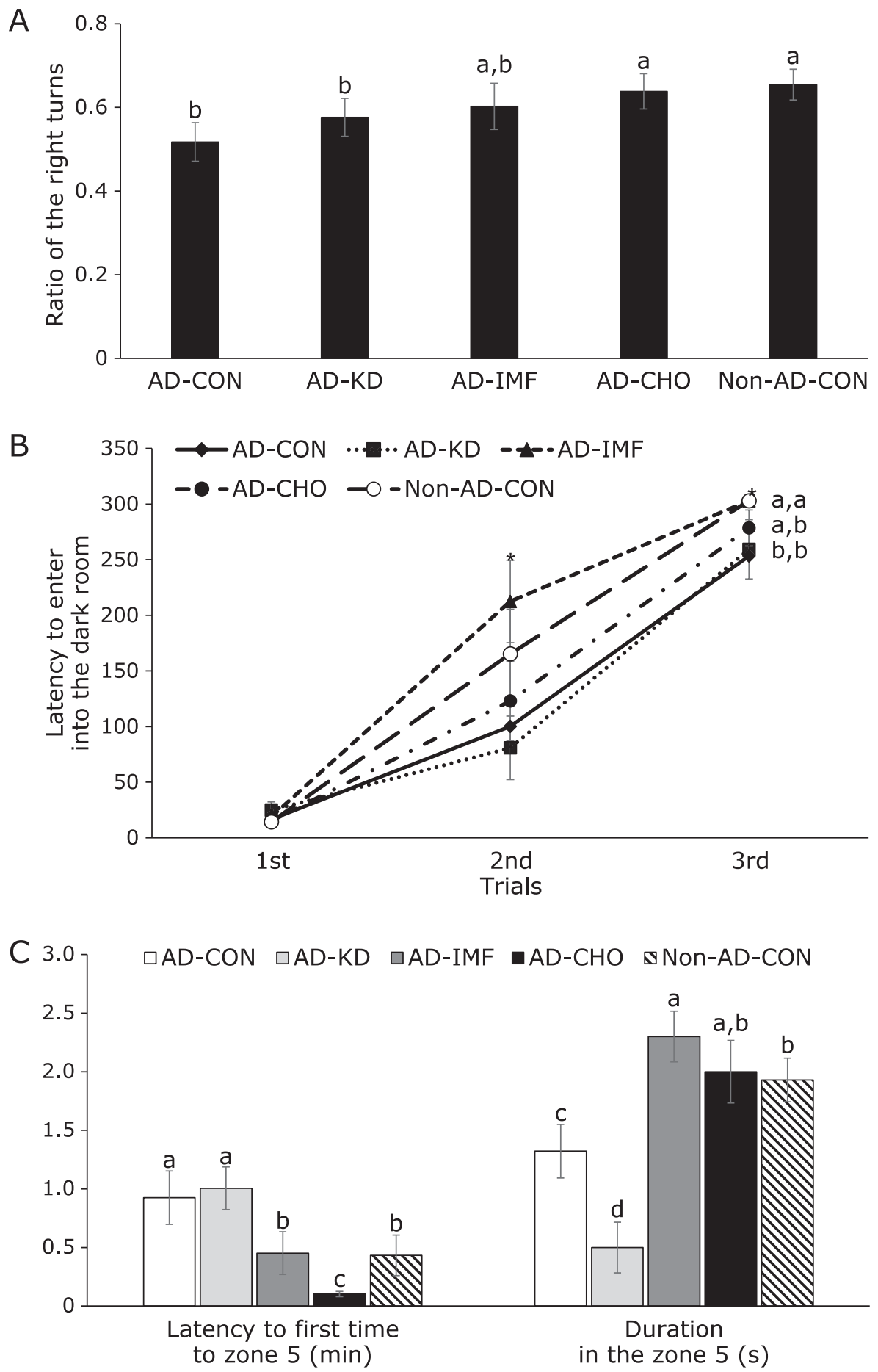

Fig. 1. Memory deficit of rats with amyloid- $\beta$ infusion. Ratio of the right turns in $Y$ maze $(A)$, latency time to enter the dark room in passive avoidance test at 3 trials (B), the latency time to locate zone with the platform and the period to stay in the platform zone on day 5 during the water maze test (C) were shown. The amyloid- $\beta(25-35)$ infused diabetic rats AD model rats received assigned diet for 8 weeks as follows: 1 ) a normal diet (28 energy \% fat diet) with ad libitum feeding (AD-CON), 2) ketogenic diet (AD-KD; 0.1\% carbohydrate diet), 3) high carbohydrate diet (AD-CHO; 68 energy \% carbohydrate diet) and 4) normal diet with intermittent fasting (AD-IMF). The sham-operated rats received the same diet (normal diet) as AD-CON (Non-AD-CON) for 8 weeks. Each dot and bar represents means \pm SD $(n=10)$. *Significantly different among the groups by one-way-ANOVA at $p<0.05$. a,b,c,d Different letters on the bars indicate significant differences by Tukey test at $p<0.05$.

in zone 5 for less time than those in the Non-AD-CON and rats, but the time in zone 5 was shortest for the AD-KD among all the groups. Rats in the AD-IMF group stayed longer in the zone 5 than those of Non-AD-CON (Fig. 1C). Rats in the AD-CHO stayed in zone 5 for a similar time as in the Non-AD-CHO. These results about memory function showed that AD-IMF improved short- and spatial-memory function the most and AD-CHO had some improvement.

Amyloid- $\beta$ deposition and brain insulin signaling. The amyloid- $\beta$ accumulation was elevated in AD-CON compared to 


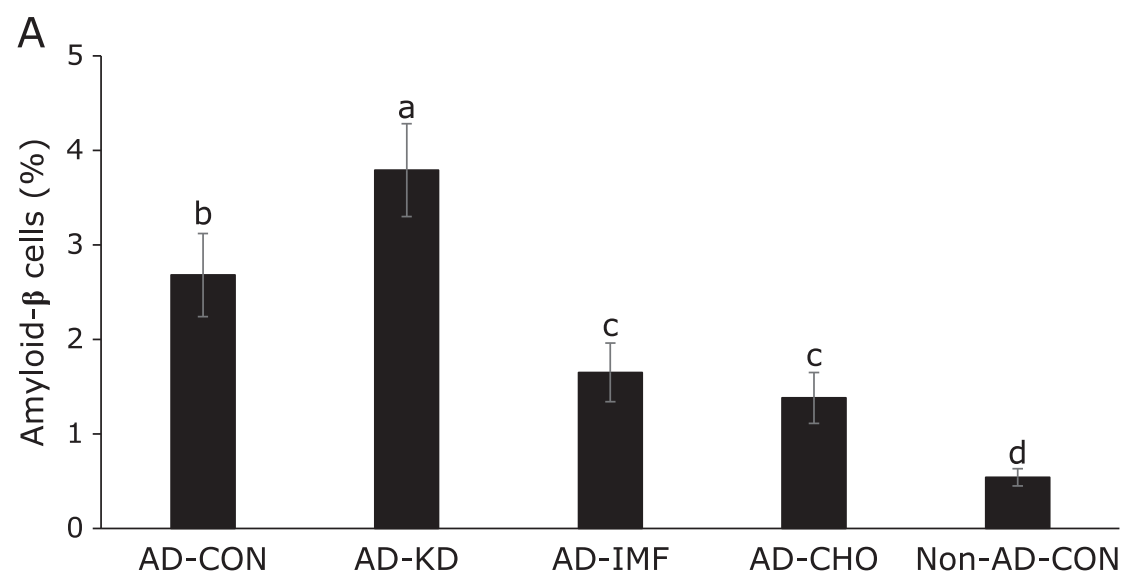

\section{$\mathrm{B}_{1.2} \square \mathrm{AD}-\mathrm{CON} \square \mathrm{AD}-\mathrm{KD} \square \mathrm{AD}-\mathrm{IMF}$ - AD-CHO $\$$ Non-AD-CON}

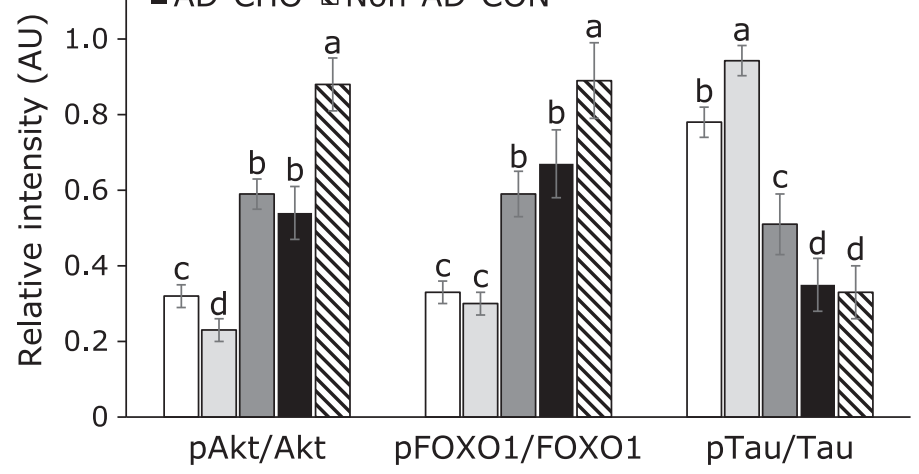

pAkt

Akt

pFOXO1

FOXO1

pTau

Tau

$\boldsymbol{\beta}$-actin

NonAD-CON AD-KD AD-IMF AD-CHO AD-CON
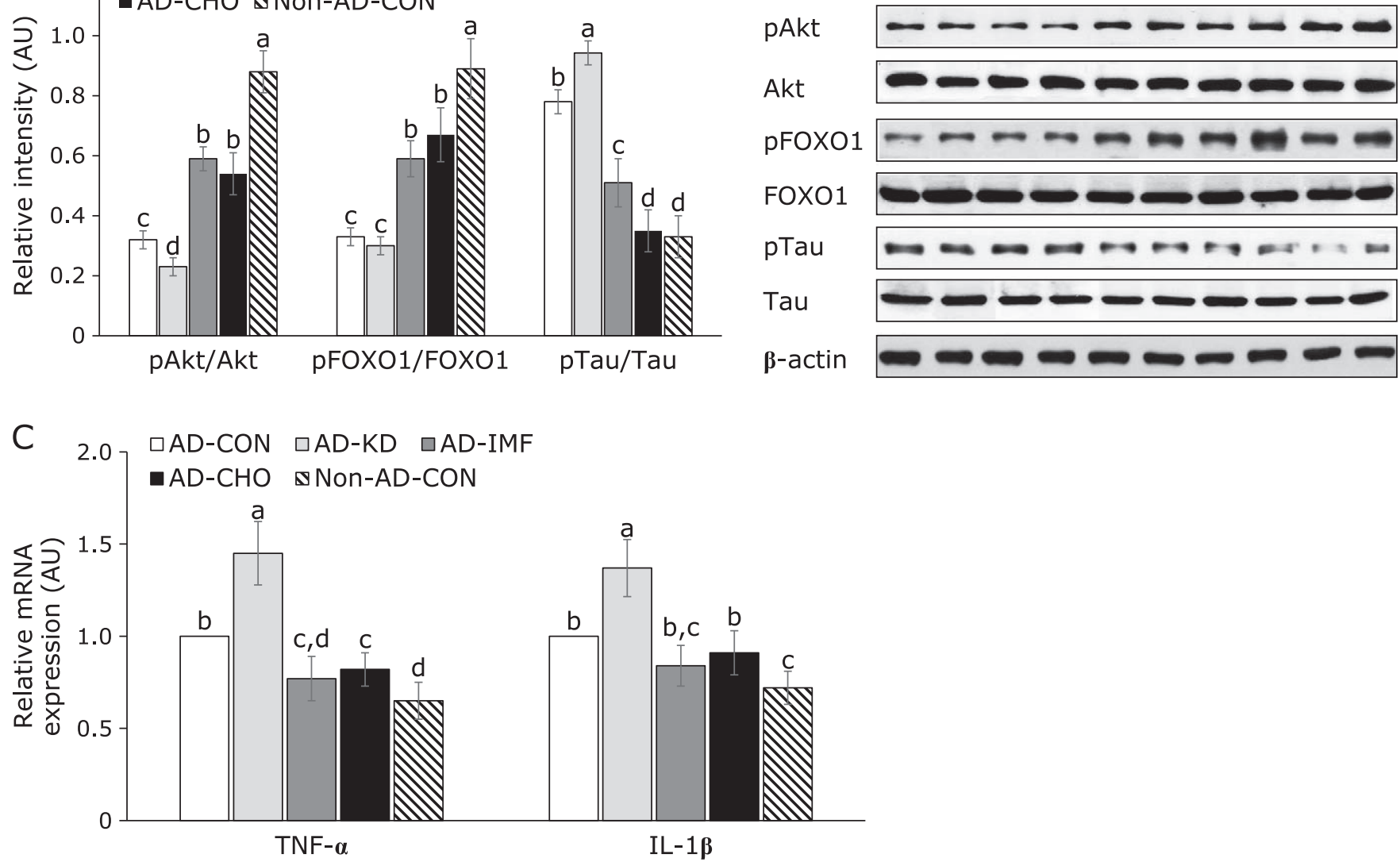

Fig. 2. Insulin signaling and cytokines in the hippocampus. After preparing lysates of the hippocampus, amyloid- $\beta$ was stained by anti-amyloid- $\beta$ antibody in brain section and the percentage of amyloid- $\beta$ stained cells $(A)$ was given $(n=5)$. The phosphorylation and expressions of proteins related to insulin signaling (B) was measured with western blot analysis and relative intensity was calculated based on designated protein contents $(n=4)$. The amyloid- $\beta(25-35)$ infused diabetic rats AD model rats received assigned diet for 8 weeks as follows: 1$)$ a normal diet $(28$ energy $\%$ fat diet) with ad libitum feeding (AD-CON), 2) ketogenic diet (AD-KD; $0.1 \%$ carbohydrate diet), 3) high carbohydrate diet (AD-CHO; 68 energy \% carbohydrate diet) and 4) normal diet with intermittent fasting (AD-IMF). The sham-operated rats received the same diet (normal diet) as AD-CON (Non-AD-CON) for 8 weeks. Each dot and bar represents means \pm SD. ${ }^{a}, \mathrm{~b}, \mathrm{c}, \mathrm{d}$ Different letters on the bars indicate significant differences by Tukey test at $p<0.05$.

Non-AD-CON and it further increased in AD-KD compared to the AD-CON. AD-IMF and AD-CHO lowered AB accumulation (Fig. 2A). The phosphorylation of Akt was much lower in the AD-CON than Non-AD-CON and AD-KD decreased the phosphorylation compared to the AD-CON. AD-IMF and AD-CHO increased the Akt phosphorylation compared to the AD-Con, but they did not increase it as much as the Non-AD-CON (Fig. 2B). The phosphorylation of FOXO-1, a downstream signal of Akt, was much lower in AD-CON than Non-AD-CON and AD-KD was similar to $\mathrm{AD}-\mathrm{CON}$. $\mathrm{AD}-\mathrm{IMF}$ and $\mathrm{AD}-\mathrm{CHO}$ elevated the phosphorylation of FOXO1 compared to the AD-CON, but they did not increase it similar to the Non-AD-CON (Fig. 2B). The 
Table 2. Body weight, energy intake, energy expenditure and inflammation index

\begin{tabular}{|c|c|c|c|c|c|}
\hline & $\begin{array}{l}\text { AD-CON } \\
(n=10)\end{array}$ & $\begin{array}{l}\text { AD-KD } \\
(n=10)\end{array}$ & $\begin{array}{l}\text { AD-IMF } \\
(n=10)\end{array}$ & $\begin{array}{l}\mathrm{AD}-\mathrm{CHO} \\
(n=10)\end{array}$ & $\begin{array}{l}\text { Non-AD-CON } \\
\quad(n=10)\end{array}$ \\
\hline Final body weight (g) & $366 \pm 27^{\mathrm{ab}}$ & $353 \pm 32^{b}$ & $291 \pm 27^{d}$ & $317 \pm 26^{c}$ & $373 \pm 15^{a}$ \\
\hline Body weight gain (g) & $169 \pm 12^{\mathrm{a}}$ & $165 \pm 15^{a}$ & $94.4 \pm 8.4^{c}$ & $117 \pm 14^{b}$ & $175 \pm 9.2^{\mathrm{a}}$ \\
\hline Epididymal fat pads/body weight (g/kg body weight) & $11.3 \pm 1.0^{\mathrm{a}}$ & $11.0 \pm 0.7^{\mathrm{a}}$ & $9.9 \pm 0.5^{\mathrm{b}}$ & $10.1 \pm 0.8^{\mathrm{b}}$ & $11.0 \pm 1.0^{\mathrm{a}}$ \\
\hline Retroperitoneal fat/body weight (g/kg body weight) & $11.1 \pm 0.7^{\mathrm{a}}$ & $11.3 \pm 0.9^{a}$ & $9.8 \pm 0.9^{b}$ & $9.2 \pm 0.7^{b}$ & $11.1 \pm 1.0^{\mathrm{a}}$ \\
\hline Visceral fat ( $\mathrm{g} / \mathrm{kg}$ body weight) & $22.4 \pm 1.6^{a}$ & $22.3 \pm 1.4^{\mathrm{a}}$ & $19.7 \pm 1.1^{\mathrm{b}}$ & $19.3 \pm 1.7^{\mathrm{b}}$ & $22.1 \pm 1.9^{a}$ \\
\hline Food intake (g/day) & $16.6 \pm 1.8^{\mathrm{a}}$ & $9.7 \pm 1.0^{\mathrm{b}}$ & $10.3 \pm 2.1^{b}$ & $15.8 \pm 1.7^{\mathrm{a}}$ & $16.7 \pm 1.6^{\mathrm{a}}$ \\
\hline Energy intake (kcal/day) & $71.3 \pm 7.7 a$ & $63.8 \pm 6.6 b$ & $44.4 \pm 9.0 c$ & $61.5 \pm 6.6 b$ & $71.6 \pm 6.9 a$ \\
\hline Energy expenditure (kcal/body weight/day) & $103 \pm 10^{\mathrm{ab}}$ & $110 \pm 11^{\mathrm{a}}$ & $117 \pm 12^{\mathrm{a}}$ & $110 \pm 11^{\mathrm{a}}$ & $94 \pm 11^{b}$ \\
\hline Respiratory quotient & $0.86 \pm 0.09^{\mathrm{ab}}$ & $0.81 \pm 0.09^{b}$ & $0.82 \pm 0.09^{\mathrm{b}}$ & $0.92 \pm 0.08^{\mathrm{a}}$ & $0.81 \pm 0.08^{b}$ \\
\hline $\begin{array}{l}\text { Carbohydrate oxidation during dark cycle } \\
\text { ( } \mathrm{mg} / \mathrm{kg} \text { body weight } / \mathrm{min} \text { ) }\end{array}$ & $5.8 \pm 0.7^{\mathrm{b}}$ & $4.1 \pm 0.7^{c}$ & $4.7 \pm 0.7^{c}$ & $8.7 \pm 0.9^{\mathrm{a}}$ & $3.5 \pm 0.5^{d}$ \\
\hline Fat oxidation during dark cycle ( $\mathrm{mg} / \mathrm{kg}$ body weight $/ \mathrm{min})$ & $5.2 \pm 0.6^{c}$ & $7.6 \pm 0.8^{\mathrm{a}}$ & $7.8 \pm 0.9^{a}$ & $3.0 \pm 0.4^{d}$ & $6.5 \pm 0.8^{b}$ \\
\hline
\end{tabular}

The amyloid- $\beta(25-35)$ infused diabetic rats $A D$ model rats received assigned diet for 8 weeks as follows: 1 ) a normal diet (28 energy $\%$ fat diet) with ad libitum feeding (AD-CON), 2) ketogenic diet (AD-KD; 0.1\% carbohydrate diet), 3) high carbohydrate diet (AD-CHO; 68 energy \% carbohydrate diet) and 4) normal diet with intermittent fasting (AD-IMF). The sham-operated rats received the same diet (normal diet) as AD-CON (Non-AD-CON)

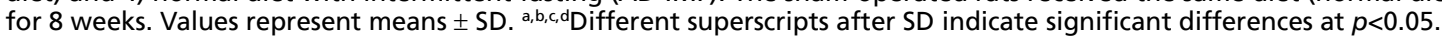

Table 3. Lipid profiles and glucose and insulin levels in the circulation of overnight-fasted rats

\begin{tabular}{|c|c|c|c|c|c|}
\hline & $\begin{array}{l}\text { AD-CON } \\
(n=10)\end{array}$ & $\begin{array}{l}\text { AD-KD } \\
(n=10)\end{array}$ & $\begin{array}{l}\text { AD-IMF } \\
(n=10)\end{array}$ & $\begin{array}{l}\mathrm{AD}-\mathrm{CHO} \\
(n=10)\end{array}$ & $\begin{array}{c}\text { Non-AD-CON } \\
(n=10)\end{array}$ \\
\hline Serum $\beta$-hydroxy butyrate (mM) & $0.15 \pm 0.05^{c}$ & $0.40 \pm 0.09^{\mathrm{a}}$ & $0.24 \pm 0.07^{b}$ & $0.14 \pm 0.07^{c}$ & $0.15 \pm 0.06^{c}$ \\
\hline Fasting serum glucose (mg/dl) & $126.3 \pm 11.4$ & $128.0 \pm 11.4$ & $122.2 \pm 15.4$ & $119.8 \pm 12.9$ & $120.1 \pm 12.1$ \\
\hline Fasting serum insulin (ng/ml) & $2.52 \pm 0.39^{b}$ & $2.51 \pm 0.28^{b}$ & $3.01 \pm 0.41^{\mathrm{a}}$ & $1.86 \pm 0.26 c$ & $2.01 \pm 0.32^{c}$ \\
\hline HOMA-IR & $14.1 \pm 1.5^{b}$ & $14.3 \pm 1.3^{b}$ & $16.6 \pm 2.1^{\mathrm{a}}$ & $9.9 \pm 1.2^{c}$ & $10.7 \pm 1.3^{c}$ \\
\hline Total cholesterol $(\mathrm{mg} / \mathrm{dl})$ & $83.9 \pm 9.1^{b}$ & $73.2 \pm 5.9^{c}$ & $91.7 \pm 9.7^{b}$ & $108 \pm 10^{\mathrm{a}}$ & $75.4 \pm 8.5^{\mathrm{bc}}$ \\
\hline HDL cholesterol (mg/dl) & $23.6 \pm 3.2^{b}$ & $30.2 \pm 3.8^{\mathrm{a}}$ & $21.9 \pm 3.2^{b}$ & $17.6 \pm 2.7^{c}$ & $24.1 \pm 3.1^{\mathrm{b}}$ \\
\hline LDL cholesterol (mg/dl) & $47.7 \pm 5.5^{c}$ & $33.2 \pm 3.9^{\mathrm{e}}$ & $60.5 \pm 6.2^{b}$ & $77.8 \pm 8.2^{\mathrm{a}}$ & $40.9 \pm 4.9^{d}$ \\
\hline Triglyceride levels (mg/dl) & $62.7 \pm 5.8^{\mathrm{a}}$ & $54.4 \pm 6.3^{b}$ & $60.2 \pm 5.6^{a}$ & $63.7 \pm 6.3^{\mathrm{a}}$ & $53.2 \pm 6.3^{b}$ \\
\hline
\end{tabular}

The amyloid- $\beta(25-35)$ infused diabetic rats $A D$ model rats received assigned diet for 8 weeks as follows: 1 ) a normal diet (28 energy \% fat diet) with ad libitum feeding (AD-CON), 2) ketogenic diet (AD-KD; $0.1 \%$ carbohydrate diet), 3) high carbohydrate diet (AD-CHO; 68 energy $\%$ carbohydrate diet) and 4) normal diet with intermittent fasting (AD-IMF). The sham-operated rats received the same diet (normal diet) as AD-CON (Non-AD-CON) for 8 weeks. Values represent means \pm SD. ${ }^{a, b, c, d}$ Different superscripts after SD indicate significant differences at $p<0.05$.

phosphorylation of tau, an indicator of amyloid- $\beta$ deposition, increased in AD-CON compared to the Non-AD-CON. AD-KD exacerbated tau phosphorylation. Tau phosphorylation was lower in $\mathrm{AD}-\mathrm{IMF}$ and $\mathrm{AD}-\mathrm{CHO}$ than $\mathrm{AD}-\mathrm{CON}$ (Fig. 2B).

Hippocampal TNF- $\alpha$ and IL-1 $\beta$ mRNA expression was higher in the AD-CON than the Non-AD-CON (Fig. 2C). Their expressions were lower in $\mathrm{AD}-\mathrm{IMF}$ and $\mathrm{AD}-\mathrm{CHO}$, but they increased in AD-IMF, compared to the AD-CON (Fig. 2C).

Energy metabolism. Body weight gain was not significantly different between AD-CON and Non-AD-CON, and AD-KD did not modulate body weight gain from the AD-KD. However, $\mathrm{AD}-\mathrm{CHO}$ and $\mathrm{AD}-\mathrm{IMF}$ significantly lowered body weight gain compared to $\mathrm{AD}-\mathrm{CON}$, and $\mathrm{AD}-\mathrm{IMF}$ decreased it more than AD-CHO (Table 2). Visceral fat mass was determined by summing the weight of retroperitoneal fat and epididymal fat mass. Visceral fat mass was not significantly different between ADCON and Non-AD-CON and its mass in the AD-KD did not differ from the AD-CON (Table 2). However, AD-IMF and AD-CHO decreased the visceral fat mass compared to the AD-CON. In visceral fat mass retroperitoneal fat and epididymal fat mass showed the similar patterns.

Weight gain and visceral fat mass are altered by energy intake and energy expenditure (Table 2). Energy intake was similar in both of AD-CON and Non-AD-CON. Energy intake in the AD$\mathrm{KD}, \mathrm{CHO}$ and $\mathrm{AD}-\mathrm{IMF}$ groups lowered energy intake compared to the AD-CON and that in the AD-IMF was lowest among the groups. Energy expenditure tended to be lower in Non-AD-CON than AD-CON $(p=0.06)$ whereas energy expenditure in the other experimental groups of the AD rats did not differ significantly from that in the AD-CON. Energy expenditure was significantly higher in Non-AD-CON than the AD-IMF, AD-CHO, and ADKD groups (Table 2). RQ did not significantly differ between AD-CON and Non-AD-CON, and it was significantly lower in the $\mathrm{AD}-\mathrm{KD}$ than $\mathrm{AD}-\mathrm{CHO}$. According to RQ, daily energy expenditure and body weight, carbohydrate, and fat oxidation as fuel sources were significantly different among the groups. Carbohydrate oxidation was significantly higher in AD-CON than Non-AD$\mathrm{CON}$ and it was lowered in the descending order of $\mathrm{AD}-\mathrm{CHO}$, $\mathrm{AD}-\mathrm{CON}$, and AD-IMF = AD-KD (Table 2). In contrast to carbohydrate oxidation, fat oxidation was higher in the Non-AD-CON than $\mathrm{AD}-\mathrm{CON}$ and among $\mathrm{AD}$ rats, it was higher in the ascending order of AD-CHO, AD-CON, and AD-IMF = AD-KD (Table 2). These results suggested that $\mathrm{AD}$ increased the utilization of carbohydrate more than fat as a fuel and that IMF and AD-KD suppressed the utilization of carbohydrate.

Glucose metabolism. Serum $\beta$-hydroxy butyrate concentrations were not significantly different between AD-CON, AD$\mathrm{CHO}$, and Non-AD-CON, but the concentrations were higher in $\mathrm{AD}-\mathrm{KD}$ and AD-IMF than AD-CHO (Table 3 ). Serum $\beta$-hydroxy butyrate concentrations in AD-KD was much higher than AD-IMF 
A
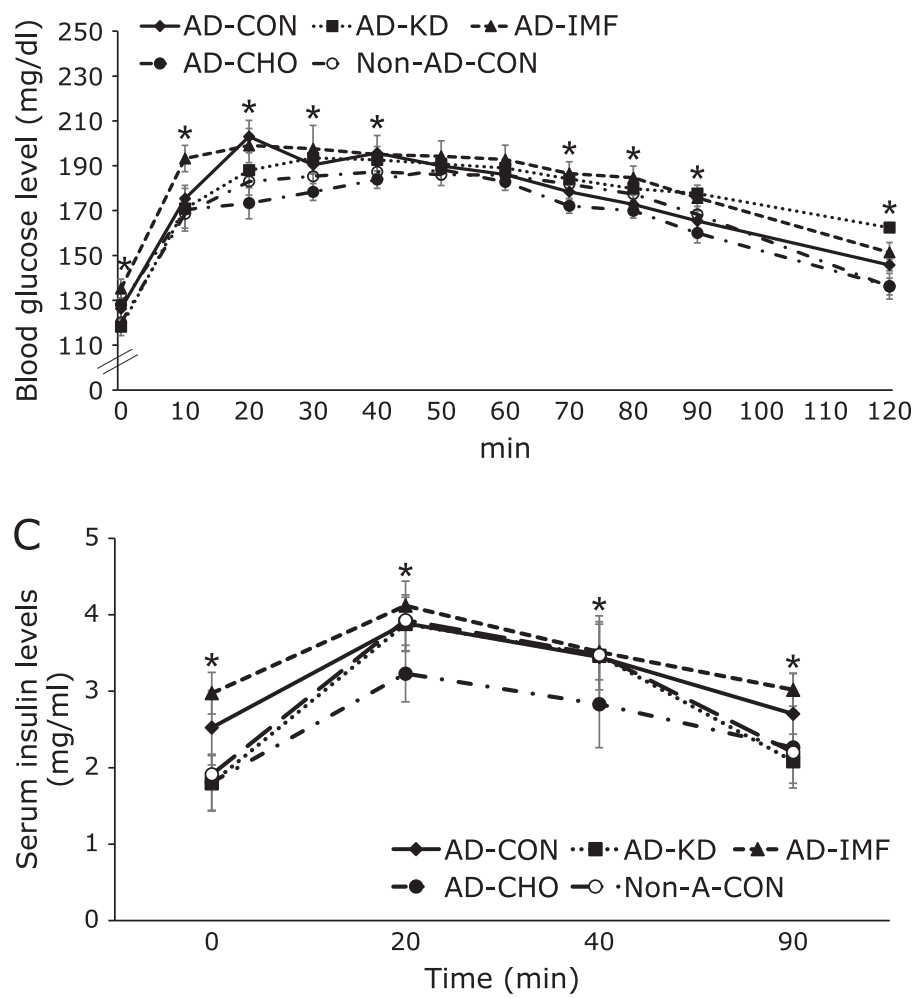

B

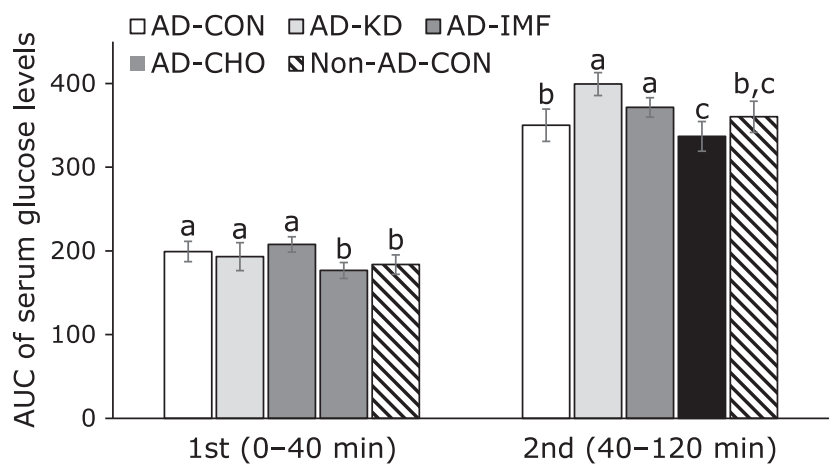

D

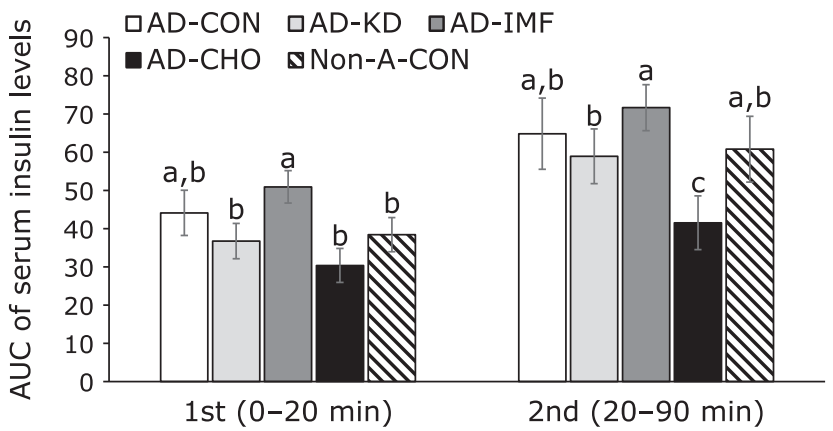

Fig. 3. Oral glucose tolerance test. Glucose ( $2 \mathrm{~g} / \mathrm{kg}$ body weight) was orally administered to the rats. Serum glucose levels (A) and serum insulin levels (C) were also measured at the assigned times after glucose challenge. Area under the curve (AUC) of glucose (B) and insulin (D) was calculated in the first ( $0-40 \mathrm{~min})$ and second phases (40-120 min). The amyloid- $\beta(25-35)$ infused diabetic rats $A D$ model rats received assigned diet for 8 weeks as follows: 1) a normal diet (28 energy \% fat diet) with ad libitum feeding (AD-CON), 2) ketogenic diet (AD-KD; 0.1\% carbohydrate diet), 3) high carbohydrate diet (AD-CHO; 68 energy \% carbohydrate diet) and 4) normal diet with intermittent fasting (AD-IMF). The sham-operated rats received the same diet (normal diet) as AD-CON (Non-AD-CON) for 8 weeks. Each dot and bar represents means \pm SD $(n=10)$. ${ }^{\circ}$ Significantly different among the groups by one-way-ANOVA at $p<0.05$. ${ }^{a, b}, c$ Different letters on the bars indicate significant differences by Tukey test at $p<0.05$.

(Table 3). Overnight-fasting serum glucose concentrations were not significantly different among the groups (Table 3 ). However, serum insulin levels at fasting state were higher in the AD-CON than Non-AD-CON, and they were higher in AD-IMF in comparison to AD-CON and AD-CHO (Table 3). As calculated by serum glucose and insulin levels at fasting state, HOMA-IR was higher in the ascending order of AD-CHO, Non-AD-CON, $\mathrm{AD}-\mathrm{CON}=\mathrm{AD}-\mathrm{KD}$, and $\mathrm{AD}-\mathrm{IMF}$ and it were similar between $\mathrm{AD}-\mathrm{CHO}$ and Non-AD-CON (Table 3).

Serum glucose levels were increased until the peak level in the AD-CON compared to the Non-AD-CON. Among different interventions, the peak levels were lower in AD-CHO than ADIMF and AD-KD (Fig. 3A). The 1st phase AUC of serum glucose levels during OGTT was much higher in the AD-CON than the Non-AD-CON and it slightly increased in the AD-IMF compared to AD-CON. The AUC at the 1st phase was similar between AD-CHO and Non-AD-CON. After 40 min during OGTT serum glucose levels were slowly decreased in all rats, but the slope of the decrease in the AUC was lower in AD-CHO than AD-KD and AD-CON (Fig. 3B).

After reaching the peak, serum glucose concentrations decreased in all rats, but the slope of decrease was different among the groups. Serum glucose levels decreased more slowly in the AD-CON than the Non-AD-CON. Among the interventions, AD-KD lowered serum glucose levels most slowly, and AD-IMF also decreased the levels more slowly than AD-CON (Fig. 3A). The area under the curve (AUC) of serum glucose levels in the 1st part was higher in the AD-CON than the Non-AD-CON, and it was lowered in descending order of AD-IMF, AD-CON, AD-KD, and $\mathrm{AD}-\mathrm{CHO}=$ Non-AD-CON. The 2 nd parts of OGTT was higher in the AD-CON than the Non-AD-CON, and it increased in $\mathrm{AD}-\mathrm{IMF}$ and $\mathrm{AD}-\mathrm{KD}$ and decreased $\mathrm{AD}-\mathrm{CHO}$ compared to the AD-CON (Fig. 3B). Serum insulin levels were not significantly different between AD-CON and Non-AD-CON and the levels at 20 and $40 \mathrm{~min}$ were similar among AD-CON, AD-KD, and AD-IMF (Fig. 3C and D). They were much lower in AD-KD than AD-CON (Fig. 3C and D).

After intraperitoneal injection of insulin, serum glucose concentration in rats in the AD-CON decreased until 30 min and lower serum glucose levels were maintained from 60-90 min compared to the Non-AD-CON, and no rebounce was detected in the NonAD-CON (Fig. 4A). Rats in the AD-KD exhibited the highest serum glucose levels during IPITT among the groups (Fig. 4A). $\mathrm{AD}-\mathrm{KD}$ decreased the response to insulin and it may induce hyperglycemia when glucose availability increases. However, serum glucose levels in the 1st part of IPITT of the AD-IMF quickly decreased until $30 \mathrm{~min}$, and then the levels were maintained in comparison to the rats with AD-CON groups (Fig. 4A). Rats in the $\mathrm{CHO}$ group exhibited rapidly decreased serum glucose levels similar to the AD-IMF (Fig. 4A). AUC at 1st and 2nd parts was greater in the AD-CON than Non-AD-CON (Fig. 4B). AUC of the 1st part of IPITT was higher in the ascending order of $\mathrm{AD}-\mathrm{CHO}$, Non-AD-CON $=\mathrm{AD}-\mathrm{IMF}$, and AD-CON = AD-KD. However, AUC of the 2nd part was much greater in AD-KD; thus rats with $\mathrm{AD}-\mathrm{IMF}$ and $\mathrm{AD}-\mathrm{CHO}$ showed lower serum glucose levels than those with AD-CON (Fig. 4B). The results suggested 
A

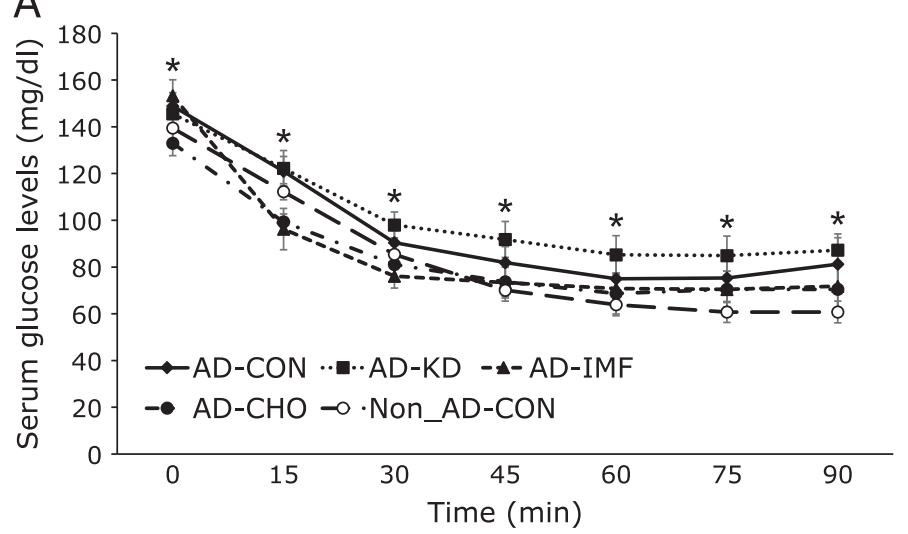

B

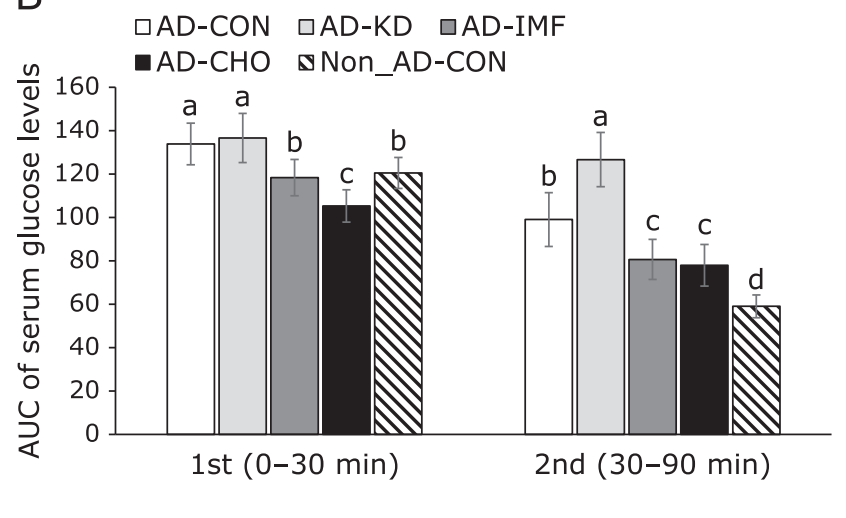

Fig. 4. Changes in the serum glucose levels during an intraperitoneal insulin tolerance test (IPITT). An IPITT was conducted by intraperitoneally injecting insulin ( $1 \mathrm{U} / \mathrm{kg}$ body weight) after a $6 \mathrm{~h}$ fast. The serum glucose levels (A) and area under the curve of serum glucose levels (B) were measured. The amyloid- $\beta(25-35)$ infused diabetic rats AD model rats received assigned diet for 8 weeks as follows: 1 ) a normal diet (28 energy $\%$ fat diet) with ad libitum feeding (AD-CON), 2) ketogenic diet (AD-KD; $0.1 \%$ carbohydrate diet), 3) high carbohydrate diet (AD-CHO; 68 energy \% carbohydrate diet) and 4) normal diet with intermittent fasting (AD-IMF). The sham-operated rats received the same diet (normal diet) as AD-CON (Non-AD-CON) for 8 weeks. Each dot and bar represents means \pm SD $(n=10)$. *Significantly different among the groups by one-way-ANOVA at $p<0.05$. ${ }^{a, b, c, d}$ Different letters on the bars indicate significant differences by Tukey test at $p<0.05$.

that $\mathrm{AD}-\mathrm{CON}$ were insulin resistant and that $\mathrm{AD}-\mathrm{IMF}$ and $\mathrm{AD}-$ $\mathrm{CHO}$ decreased the insulin resistance at high serum glucose levels when insulin was high.

Lipid metabolism. Serum total and HDL cholesterol levels did not differ between the AD-CHO and Non-AD-CON but serum LDL and triglyceride concentrations were higher in the AD-CON than the Non-AD-CON (Table 3). Interestingly, AD-KD lowered serum total and LDL concentrations and triglyceride concentrations and increased HDL concentrations in comparison to the AD-CON (Table 3). However, AD-CHO increased total and LDL cholesterol and triglyceride concentrations compared to the AD-CON, and it decreased serum HDL cholesterol concentrations (Table 3). AD-IMF did not alter lipid profiles in the circulation compared to the control. The results suggested that AD-CON rats exhibited some dyslipidemia and that $\mathrm{AD}-\mathrm{CHO}$ worsened cholesterol metabolism and AD-KD rather improved it (Table 3 ).

Gut microbiome. The number of bacterial species did not differ between AD-CON and Non-AD-CON whereas AD-CHO tended to increase the number of species $(p=0.063)$ and AD-KD significantly decreased it. The Shannon index, an index of the richness of gut microbiome, showed the same tendency as the number of species. Shannon index was much lower in AD-KD than AD-CON (Fig. 5A). Principal coordinate analysis (PCoA) shows the clustering of the gut bacterial community in the groups, and there was a significant difference among the groups $(p=0.012$; Fig. 5B). The AD-CON group exhibited a separation of the community from the Non-AD-CON with exception of one group. AD-CHO showed a separating cluster form AD-CON which was shared with Non-AD-CON. AD-IMF had sharing with AD-CON and Non-AD-CON. AD-KD had a separated cluster with NonAD-CON and AD-CON (Fig. 5B). Thus, AD-CON somewhat changed the gut bacterial community from Non-AD-CON and $\mathrm{AD}-\mathrm{KD}$ and $\mathrm{AD}-\mathrm{CHO}$ influenced the gut microbiome in AD rats.

The significant differences of the gut microbiota community among all groups were examined by analysis of molecular variance $(p=0.022)$. At the Order level, the relative counts of Clostridales were higher in AD-Con than Non-AD-CON, but those of Bacteroidales were not significantly different between the two groups (Fig. 5C). AD-IMF decreased Clostridales and had a tendency to increase Lactobacillales. However, AD-KD increased the relative counts of Proteobacteria especially Enterobacteriales compared to other groups (Fig. 5C).

\section{Discussion}

Ketones are produced as a consequence of low carbohydrate diets, intermittent fasting, and very low calorie diets. However, the interventions using ketogenic diet and intermittent fasting may have similar or different effects on memory function when they produce ketones. Ketone utilization can reduce the production of reactive oxygen species by modulating the ratio of oxidized and reduced form of NAD to improve mitochondrial function. ${ }^{(27)}$ We hypothesized that dietary interventions that increase ketone production may modulate mild cognitive impairment, glucose metabolism, inflammation, and the gut microbiome in amyloid- $\beta$ infused rats, and the mechanisms involved were explored. A high $\mathrm{CHO}$ diet was also included since it is an opposite intervention from the ketogenic diet. Hippocampal insulin resistance and neuroinflammation were evaluated in the present study studied since they are known to impair memory function. AD-KD and AD-IMF differently influenced memory function, although both increased serum ketone levels. AD-IMF improved memory function and but AD-KD did not protect against memory impairment. AD-IMF potentiated hippocampal insulin signaling (pAkt $\rightarrow$ pGSK-3 $\beta$ ) and reduced mRNA expression of TNF- $\alpha$ and IL-1 $\beta$, inflammatory markers, in the hippocampus but AD-KD rather impaired them. The changes of insulin sensitivity and inflammation are the brain is known to be associated with gut microbiome. $\mathrm{AD}-\mathrm{KD}$ induced gut dysbiosis compared to the Non-AD-CON and AD-IMF did not alter gut microbiome from the Non-AD-CON: AD-KD disrupted gut microbiome composition by increasing Proteobacteria and lowering diversity, but AD-IMF improved it. AD-CHO had an overlapped cluster of the gut microbiome with Non-AD-CON and it enhanced memory function. Therefore, the AD-KD increased amyloid- $\beta$ in the hippocampus more than the AD-CON by attenuating hippocampal insulin signaling and increasing neuroinflammation. The AD-IMF and AD-CHO prevented the attenuation of insulin signaling and inflammation in the hippocampus and the prevention of gut dysbiosis might be involved in the process. The IMF and CHO diet patterns might be beneficial for protecting against the development of Alzheimer's disease.

The ketogenic diet is a very low-carbohydrate and high-fat diet that mimics the fasting state. It is known to have a beneficial effects on epileptic seizures that develop due to abnormally exces- 
A

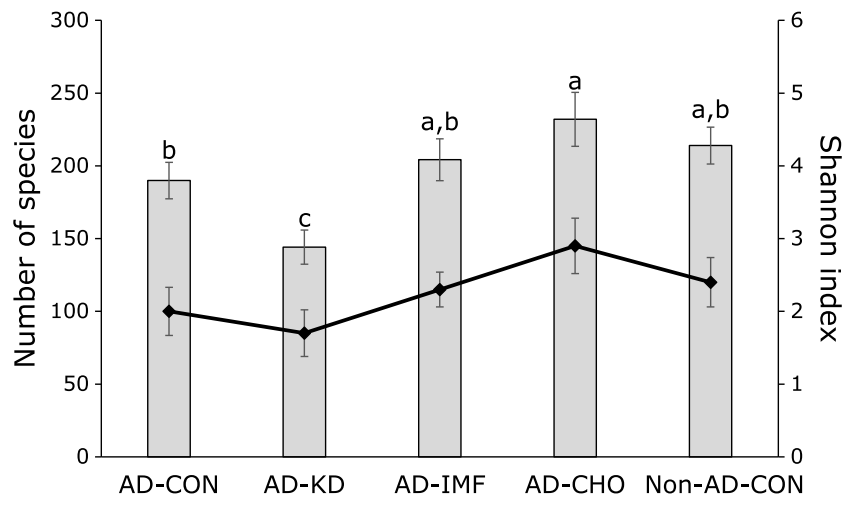

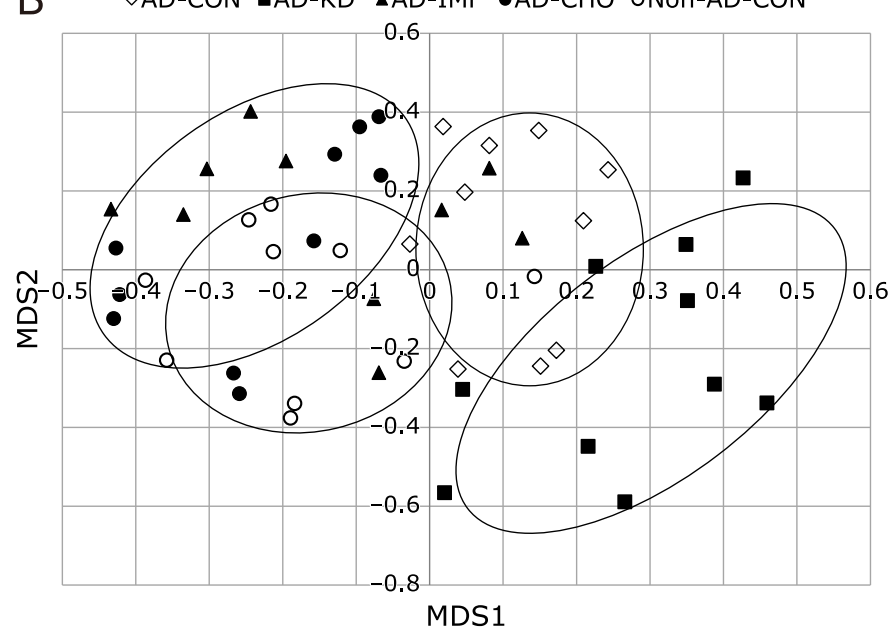

C

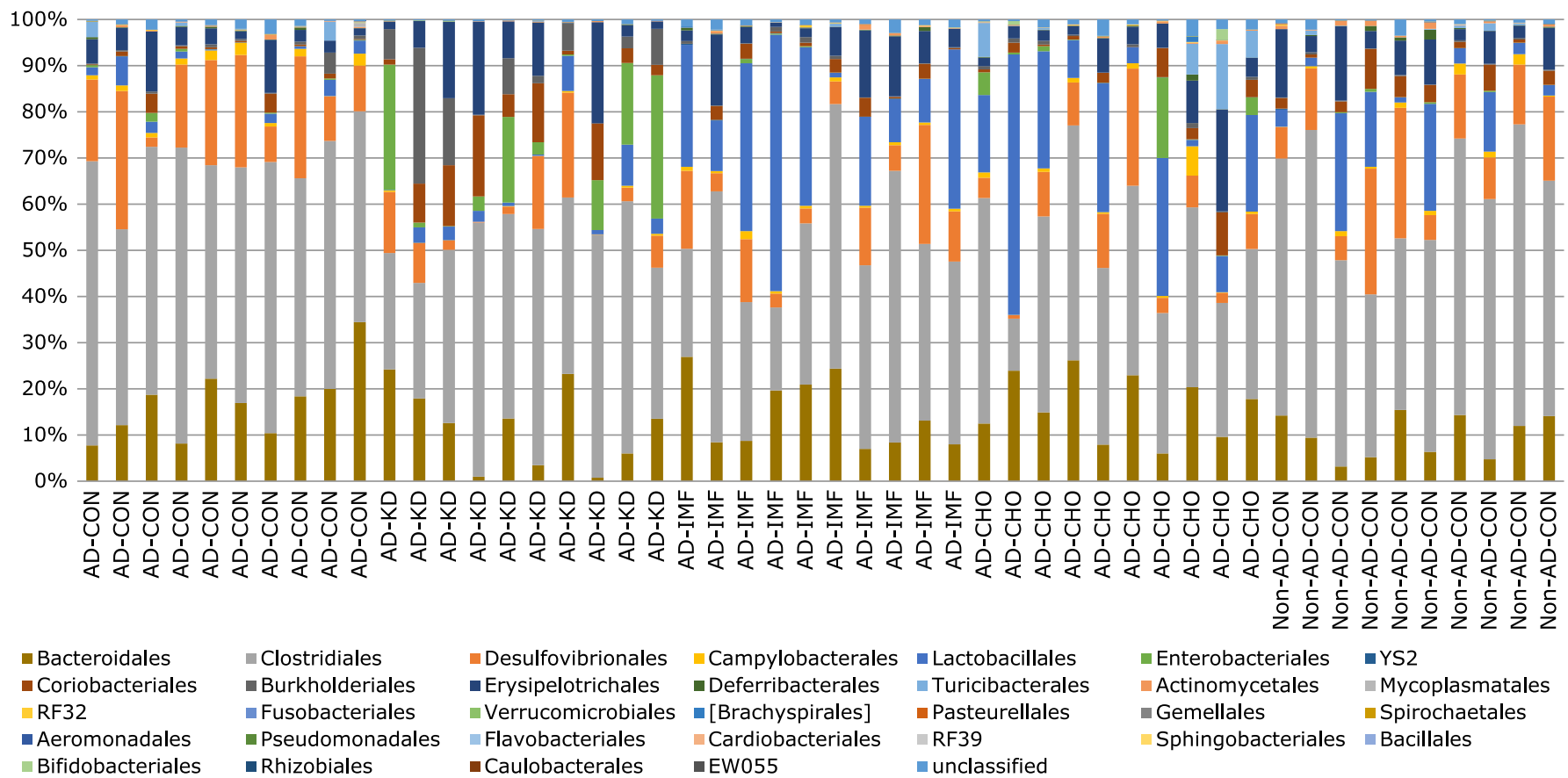

Fig. 5. The profiles of gut microbiomes. Feces were collected from the cecum at the end of experimental periods and the bacterial DNA was analyzed. The number of species and Shannon index (A), the fecal bacterial community by principal coordinate analysis (PCOA) (B) and proportion of taxonomic assignments [order] for gut microbiomes $(C)$ was determined from the feces. The amyloid- $\beta(25-35)$ infused diabetic rats $A D$ model rats received assigned diet for 8 weeks as follows: 1 ) a normal diet (28 energy $\%$ fat diet) with ad libitum feeding (AD-CON), 2) ketogenic diet (AD-KD; $0.1 \%$ carbohydrate diet), 3) high carbohydrate diet (AD-CHO; 68 energy \% carbohydrate diet) and 4) normal diet with intermittent fasting (AD-IMF). The sham-operated rats received the same diet (normal diet) as AD-CON (Non-AD-CON) for 8 weeks. Each dot and bar represents means \pm SD $(n=10)$. a,b, Different letters on the bars indicate significant differences by Tukey test at $p<0.05$.

sive neuronal activity in the brain. ${ }^{(28)}$ The seizures are accompanied by uncontrolled shaking movements. KD reduces the symptoms of seizure by using ketone instead of glucose in the brain. The KD effects on cognitive function and brain growth have been researched, but the results are contradictory. The KD was shown to have harmful effects on memory function and brain growth, ${ }^{(29)}$ but another study demonstrated beneficial effects on brain function. ${ }^{(30)}$ Ketones, acetoacetate, and $\beta$-hydroxy butyrate are alternative energy sources to glucose. Persons with Alzheimer's disease have an impairment of glucose uptake in the brain, but ketone utilization in the brain appears not to be impaired. ${ }^{(31)}$ The different results may be associated with types of fat utilized in the ketogenic diet. Medium-chain fat intake has better effects on epilepsy and neurodegenerative diseases. However, intermittent fasting has been reported to improve memory function, although both the ketogenic diet and intermittent fasting elevate serum ketone levels by producing ketones in the liver.

The main characteristics of Alzheimer's disease are amyloid- $\beta$ deposition in the hippocampus and impairment of memory function. ${ }^{(32)}$ The mechanism of amyloid- $\beta$ accumulation in the hippocampus remains unclear. The changes of amyloid precursors into amyloid- $\beta$ and tangles of amyloid- $\beta$ in the hippocampus are 
involved in the development and progression of Alzheimer's diseases. ${ }^{(32)}$ The major cause is the attenuation of hippocampal insulin signaling and potentiation of tau phosphorylation. ${ }^{(33)}$ The brain insulin sensitivity was partly consistent with peripheral insulin sensitivity. ${ }^{(34)} \mathrm{IMF}$, but not KD, improved systemic insulin sensitivity measured by IPITT but HOMA-IR, representing insulin resistance in the fasting state, was higher in AD-IMF than AD-KD in the present study. Furthermore, hippocampal insulin signaling and neuroinflammation are reported to be crucial to preventing and alleviating amyloid- $\beta$ deposition in an animal model using infused amyloid- $\beta$ into the hippocampus. ${ }^{(24,35,36)}$ AD-IMF improved hippocampal insulin signaling and neuroinflammation but its ketone production was not as much as AD-KD in the present study. This present study revealed that AD-IMF and AD-CHO potentiated insulin signaling and reduced neuroinflammation in the present study but not AD-KD. The lack of benefit appeared to be related to its effects on the gut microbiome. AD-KD induced gut dysbiosis, which may have increased systemic and neuronal inflammation as shown in previous studies. ${ }^{(37,38)}$ Therefore, ketone production may help provide energy, but it also may impair insulin signaling and neuroinflammation in the hippocampus and increase the amyloid- $\beta$ deposition in the hippocampus. Improvements in insulin sensitivity and inflammation in the brain and peripheral tissues protect against memory dysfunction.

The gut microbiome is another factor that influences various neurological diseases through the bidirectional microbiota-gutbrain axis. ${ }^{(19)}$ The gut microbiota influence host memory function and neurodegenerative diseases. The gut microbiome is altered in patients with Alzheimer's disease. ${ }^{(39,40)}$ They have less Bacteroidetes and Bifidobacterium, and more Firmicutes than healthy controls but some studies have shown different results. ${ }^{(39,40)}$ Gut microbiota dysbiosis increases the permeability of both the gut and bloodbrain barrier, and they secrete large amounts of amyloids and lipopolysaccharides that contribute to the production of proinflammatory cytokines. ${ }^{(41)}$ Dysbiosis may mediate the pathogenesis of Alzheimer's disease and other neurodegenerative disorders by inflammation driven pathogenesis. ${ }^{(38)}$ The present study demonstrated that rats that developed AD-like pathologies had gut microbiome dysbiosis as evidenced by decreased relative counts of Lactobacillales, but without altering Bacteriodetes. Therefore, patients with AD may also have dysbiosis which induces neuroinflammation which in turn exacerbates memory dysfunction.

The KD has been used for modulating epilepsy treatment. ${ }^{(19)}$ $\mathrm{KD}$ decreases the relative abundance of Bifidobacterium longum by 3.4 folds and B. adolescentis by 16 folds, Eubacterium rectale by 5 folds and Dialister by 5.5 folds and increases the relative abundance of Escherichia coli by 2.7 folds in children with severe epilepsy. ${ }^{(19)} \mathrm{KD}$ diminished the relative abundance of health-promoting and fiber-consuming bacteria. KD effects on the gut microbiome remains unclear. A KD containing $4.8 \%$ fiber and $3.2 \%$ carbohydrates increased the relative abundance of putatively beneficial gut microbiota (Akkermansia muciniphila and Lactobacillus) and reduced that of putatively pro-inflammatory taxa (Desulfovibrio and Turicibacter) in young healthy mice. ${ }^{(18)}$ The present study demonstrated that KD increased the relative counts of Proteobacteria, especially Enterobacteriales whereas IMF decreased Clostridales and had a tendency to increase Bacteroidales. The KD was included no $\mathrm{CHO}, 3.4 \%$ cellulose and lard for fat source in the present study. Thus, the composition of KD might affect the changes of gut microbiome.

High $\mathrm{CHO}$ diets lead to metabolic syndrome, especially dyslipidemia in Asians who have consumed very high $\mathrm{CHO}$ and very low fat diets. ${ }^{(42)}$ However, high $\mathrm{CHO}$ diets improved memory function better than KD in the present study. Furthermore, AD-CHO improved memory function better than AD-KD and AD-CON. AD-CHO shared the bacterial community with NonAD-CON and AD-IMF. AD-CHO increased Lactobacillales and decreased Clostridales similar to AD-IMF. Thus, carbohydrate in the diet may be utilized by gut microbiota, although it is known to be mostly absorbed in the small intestine. Starch in the diet may act as a resistant starch in the animal study since high $\mathrm{CHO}$ diet acts as an anti-obesity food compared to a high fat diet. ${ }^{(43)}$ However, no study has examined a high $\mathrm{CHO}$ diet (mainly high in starch) on the gut microbiome, although a high fat diet induced microbiome dysbiosis. ${ }^{(44,45)}$ Microbiome available carbohydrates including resistant starch and soluble dietary fiber improve the gut microbiome composition. ${ }^{(46)}$ A high carbohydrate diet may influence gut microbiome possibly by providing some carbohydrate to the gut microbiota in rats.

There are some limitations to this study, first animal models are not fully applicable to humans. Additionally, although the AD model used in this study mimics human AD in many respects, the etiology of the disease is different in the rat model than in humans. In this study, the animal model was generated by the injection of amyloid- $\beta$, not by spontaneously making amyloid- $\beta$ in the brain. The deposition of amyloid- $\beta$ tangles in the hippocampus was modulated by different diets. Finally, the diets used were semisynthetic diets that are different from eating whole foods and may have different effects on the microbiome. However, despite the limitations of the study, the results suggest ways of preventing or delaying human $\mathrm{AD}$ that should be studied in humans.

In conclusion, ketone production itself might not improve memory function and the gut microbiome. KD impaired memory function compared the AD-CON, and it increased the ratio of Proteobacteria, especially Enterobacteriales, which are relatively harmful bacteria. KD needs to include sufficient microbiome available carbohydrates and optimal fat composition by modifying fat types and including non-digestible carbohydrates should be considered. IMF improved memory function and gut microbiomes, although it contained the same amounts of cellulose. Therefore, IMF and high CHO diets might improve memory function and energy and glucose metabolism. Intermittent fasting might be conducted by having long over-night fasting (16-17 h fasting) after consuming late lunch (about 3-4 PM). It can be applicable in clinical practices. It might be adopted by nursing homes, assisted living facilities, and programs like "Meals on Wheels" which provide meals for elderly people living at home.

\section{Author Contributions}

SP conceived of the study, contributed to the design and prepared the initial draft of the manuscript. TZ and JYQ carried out animal study and the statistical analysis, and TZ and XW biochemical assays. All authors read, made suggestions and approved the final version. All authors discussed the final results and approved the final manuscript.

\section{Acknowledgments}

This study was funded by a grant from the National Research Foundation of Korea (NRF) funded by the Ministry of Science and ICT (NRF-2019R1A2C1007203).

\section{Conflict of Interest}

No potential conflicts of interest were disclosed. 


\section{References}

1 Reitz C, Mayeux R. Alzheimer disease: epidemiology, diagnostic criteria, risk factors and biomarkers. Biochem Pharmacol 2014; 88: 640-651.

2 Ryu JC, Zimmer ER, Rosa-Neto P, Yoon SO. Consequences of metabolic disruption in Alzheimer's disease pathology. Neurotherapeutics 2019; 16: 600-610.

3 Gabbouj S, Ryhänen S, Marttinen M, et al. Altered insulin signaling in Alzheimer's disease brain - special emphasis on PI3K-Akt pathway. Front Neurosci 2019; 13: 629 .

4 Calsolaro V, Edison P. Neuroinflammation in Alzheimer's disease: current evidence and future directions. Alzheimers Dement 2016; 12: 719-732.

5 Akhter F, Chen D, Yan SF, Yan SS. Mitochondrial perturbation in Alzheimer's disease and diabetes. Prog Mol Biol Transl Sci 2017; 146: 341-361.

$6 \mathrm{Lu} \mathrm{J}$, Guo P, Liu X, et al. Herbal formula fo shou san attenuates Alzheimer's disease-related pathologies via the gut-liver-brain axis in APP/PS1 mouse model of Alzheimer's disease. Evid Based Complement Alternat Med 2019; 2019: 8302950 .

7 Pugazhenthi S, Qin L, Reddy PH. Common neurodegenerative pathways in obesity, diabetes, and Alzheimer's disease. Biochim Biophys Acta Mol Basis Dis 2017; 1863: 1037-1045.

8 Vasconcelos AR, Yshii LM, Viel TA, et al. Intermittent fasting attenuates lipopolysaccharide-induced neuroinflammation and memory impairment. $J$ Neuroinflammation 2014; 11: 85.

9 Seimon RV, Roekenes JA, Zibellini J, et al. Do intermittent diets provide physiological benefits over continuous diets for weight loss? A systematic review of clinical trials. Mol Cell Endocrinol 2015; 418 Pt 2: 153-172.

10 Gotthardt JD, Verpeut JL, Yeomans BL, et al. Intermittent fasting promotes fat loss with lean mass retention, increased hypothalamic norepinephrine content, and increased neuropeptide $\mathrm{Y}$ gene expression in diet-induced obese male mice. Endocrinology 2016; 157: 679-691.

11 Croteau E, Castellano CA, Richard MA, et al. Ketogenic medium chain triglycerides increase brain energy metabolism in Alzheimer's disease. $J$ Alzheimers Dis 2018; 64: 551-561.

12 Park S, Kim DS, Kang S, Daily JW 3rd. A ketogenic diet impairs energy and glucose homeostasis by the attenuation of hypothalamic leptin signaling and hepatic insulin signaling in a rat model of non-obese type 2 diabetes. Exp Biol Med (Maywood) 2011; 236: 194-204.

13 Park S, Yoo KM, Hyun JS, Kang S. Intermittent fasting reduces body fat but exacerbates hepatic insulin resistance in young rats regardless of high protein and fat diets. J Nutr Biochem 2017; 40: 14-22.

14 Shin BK, Kang S, Kim DS, Park S. Intermittent fasting protects against the deterioration of cognitive function, energy metabolism and dyslipidemia in Alzheimer's disease-induced estrogen deficient rats. Exp Biol Med (Maywood) 2018; 243: 334-343.

15 Angelucci F, Cechova K, Amlerova J, Hort J. Antibiotics, gut microbiota, and Alzheimer's disease. J Neuroinflammation 2019; 16: 108.

16 Tingirikari JMR. Microbiota-accessible pectic poly- and oligosaccharides in gut health. Food Funct 2018; 9: 5059-5073.

17 Perry RJ, Peng L, Barry NA, et al. Acetate mediates a microbiome-brain- $\beta$ cell axis to promote metabolic syndrome. Nature 2016; 534: 213-217.

18 Ma D, Wang AC, Parikh I, et al. Ketogenic diet enhances neurovascular function with altered gut microbiome in young healthy mice. Sci Rep 2018; 8: 6670 .

19 Lindefeldt M, Eng A, Darban H, et al. The ketogenic diet influences taxonomic and functional composition of the gut microbiota in children with severe epilepsy. NPJ Biofilms Microbiomes 2019; 5: 5.

20 Liu P, Jing Y, Collie ND, Campbell SA, Zhang H. Pre-aggregated A $\beta(25-35)$ alters arginine metabolism in the rat hippocampus and prefrontal cortex. Neuroscience 2011; 193: 269-282.

21 Park S, Kim DS, Kang S, Kim HJ. The combination of luteolin and Ltheanine improved Alzheimer disease-like symptoms by potentiating hippocampal insulin signaling and decreasing neuroinflammation and norepinephrine degradation in amyloid- $\beta$-infused rats. Nutr Res 2018; 60: 116-131.

22 Yang HJ, Hwang JT, Kwon DY, et al. Yuzu extract prevents cognitive decline and impaired glucose homeostasis in $\beta$-amyloid-infused rats. $J$ Nutr 2013; 143: 1093-1099.

23 Reeves PG, Nielsen FH, Fahey GC, Jr. AIN-93 purified diets for laboratory rodents: final report of the American Institute of Nutrition ad hoc writing committee on the reformulation of the AIN-76A rodent diet. J Nutr 1993; 123: 1939-1951.

24 Park S, Kang S, Kim DS, Moon BR. Agrimonia pilosa Ledeb., Cinnamomum cassia Blume, and Lonicera japonica Thunb. protect against cognitive dysfunction and energy and glucose dysregulation by reducing neuroinflam- mation and hippocampal insulin resistance in $\beta$-amyloid-infused rats. Nutr Neurosci 2017; 20: 77-88.

25 Livak KJ, Schmittgen TD. Analysis of relative gene expression data using real-time quantitative PCR and the $2^{-\Delta \Delta C_{\mathrm{T}}}$ method. Methods 2001; 25: 402-408.

26 Park S, Kim DS, Kang ES, Kim DB, Kang S. Low dose brain estrogen prevents menopausal syndrome while maintaining the diversity of the gut microbiomes in estrogen-deficient rats. Am J Physiol Endocrinol Metab 2018; 315: E99-E109.

27 Pinto A, Bonucci A, Maggi E, Corsi M, Businaro R. Anti-oxidant and antiinflammatory activity of ketogenic diet: new perspectives for neuroprotection in Alzheimer's disease. Antioxidants (Basel) 2018; 7. pii: E63.

28 Maalouf M, Rho JM, Mattson MP. The neuroprotective properties of calorie restriction, the ketogenic diet, and ketone bodies. Brain Res Rev 2009; 59: 293-315.

29 Zhao Q, Stafstrom CE, Fu DD, Hu Y, Holmes GL. Detrimental effects of the ketogenic diet on cognitive function in rats. Pediatr Res 2004; 55: 498-506.

30 Newman JC, Covarrubias AJ, Zhao M, et al. Ketogenic diet reduces midlife mortality and improves memory in aging mice. Cell Metab 2017; 26: 547557.e8

31 Cunnane SC, Courchesne-Loyer A, St-Pierre V, et al. Can ketones compensate for deteriorating brain glucose uptake during aging? Implications for the risk and treatment of Alzheimer's disease. Ann N Y Acad Sci 2016; 1367: 12-20.

32 Bischof GN, Jacobs HIL. Subthreshold amyloid and its biological and clinical meaning: long way ahead. Neurology 2019; 93: 72-79.

33 Hanseeuw BJ, Betensky RA, Jacobs HIL, et al. Association of amyloid and tau with cognition in preclinical Alzheimer disease: a longitudinal study. JAMA Neurol 2019. DOI: 10.1001/jamaneurol.2019.1424.

34 Grillo CA, Woodruff JL, Macht VA, Reagan LP. Insulin resistance and hippocampal dysfunction: disentangling peripheral and brain causes from consequences. Exp Neurol 2019; 318: 71-77.

35 Latta CH, Sudduth TL, Weekman EM, et al. Determining the role of IL-4 induced neuroinflammation in microglial activity and amyloid-beta using BV2 microglial cells and APP/PS1 transgenic mice. $J$ Neuroinflammation 2015; 12: 41.

36 Chen W, Liang T, Zuo W, et al. Neuroprotective effect of 1-deoxynojirimycin on cognitive impairment, $\beta$-amyloid deposition, and neuroinflammation in the SAMP8 mice. Biomed Pharmacother 2018; 106: 92-97.

37 Lin L, Zheng LJ, Zhang LJ. Neuroinflammation, gut microbiome, and Alzheimer's disease. Mol Neurobiol 2018; 55: 8243-8250.

38 Sochocka M, Donskow-Lysoniewska K, Diniz BS, Kurpas D, Brzozowska E, Leszek J. The gut microbiome alterations and inflammation-driven pathogenesis of Alzheimer's disease-a critical review. Mol Neurobiol 2019; 56: 1841-1851.

39 Vogt NM, Kerby RL, Dill-McFarland KA, et al. Gut microbiome alterations in Alzheimer's disease. Sci Rep 2017; 7: 13537.

40 Zhuang ZQ, Shen LL, Li WW, et al. Gut microbiota is altered in patients with Alzheimer's disease. J Alzheimers Dis 2018; 63: 1337-1346.

41 Jiang C, Li G, Huang P, Liu Z, Zhao B. The gut microbiota and Alzheimer's disease. J Alzheimers Dis 2017; 58: 1-15.

42 Park S, Ahn J, Lee BK. Very-low-fat diets may be associated with increased risk of metabolic syndrome in the adult population. Clin Nutr 2016; 35: 11591167.

43 Park S, Kim DS, Kang S. Gastrodia elata Blume water extracts improve insulin resistance by decreasing body fat in diet-induced obese rats: vanillin and 4-hydroxybenzaldehyde are the bioactive candidates. Eur J Nutr 2011; 50: $107-118$.

44 Al-Daihan S, Ben Bacha A, Al-Dbass AM, Alonazi MA, Bhat RS. High-fat diet stimulates the gut pathogenic microbiota and maintains hepatic injury in antibiotic-treated rats. Cell Mol Biol (Noisy-le-grand) 2018; 64: 103-106.

45 Shang W, Si X, Zhou Z, Li Y, Strappe P, Blanchard C. Characterization of fecal fat composition and gut derived fecal microbiota in high-fat diet fed rats following intervention with chito-oligosaccharide and resistant starch complexes. Food Funct 2017; 8: 4374-4383.

46 Maier TV, Lucio M, Lee LH, et al. Impact of dietary resistant starch on the human gut microbiome, metaproteome, and metabolome. mBio 2017; 8. pii: e01343-17.

This is an open access article distributed under the terms of the Creative Commons Attribution License, which permits unrestricted use, distribution, and reproduction in any medium, provided the original work is properly cited. 